\title{
Reaction mechanism of two-neutron transfer in DWBA
}

\author{
G. Potel ${ }^{1, \mathrm{a}}$, A. Idini ${ }^{2,3}$, F. Barranco ${ }^{4}$, E. Vigezzi ${ }^{3}$, and R.A. Broglia ${ }^{2,3,5}$ \\ 1 Departamento de Fisica Atomica, Molecular y Nuclear, Universidad de Sevilla, Facultad de Fisica, Avda. Reina Mercedes \\ s/n, Spain. \\ 2 Dipartimento di Fisica, Università di Milano, Via Celoria 16, 20133 Milano, Italy. \\ 3 INFN, Sezione di Milano Via Celoria 16, 20133 Milano, Italy. \\ 4 Departamento de Fisica Aplicada III, Universidad de Sevilla, Escuela Superior de Ingenieros, Sevilla, 41092 Camino de \\ los Descubrimientos s/n, Spain. \\ 5 The Niels Bohr Institute, University of Copenhagen, Blegdamsvej 17, 2100 Copenhagen $\emptyset$, Denmark.
}

\begin{abstract}
We present a brief introduction to the second order DWBA reaction formalism which we have used to perform the theoretical analysis of two-nucleon transfer reactions induced both by heavy and light ions. We also show an example of such a calculation, emphasizing the connection between the structure aspects of the problem and the resulting predicted two-neutron transfer cross section. The calculations were carried out making use of software specifically developed for this purpose. It includes sequential, simultaneous and non-orthogonality contributions to the process. Microscopic form factors are used which take into account the relevant structure aspects of the process, such as the nature of the single-particle wavefunctions, the spectroscopic factors, and the interaction potential responsible for the transfer. Overall agreement with the experimental absolute values of the differential cross section is obtained without any free parameter.
\end{abstract}

\section{Introduction}

Arguably, the greatest achievement of many-body physics in the fifties was that of developing the tools for a complete description and a thorough understanding of superconductivity in metals. At the basis of it one finds BCS theory and the Josephson effect. The first recognized the central role played by the appearance of a macroscopic coherent field usually viewed as a condensate of strongly overlapping Cooper pairs, the quasiparticle vacuum. The second made it clear that a true gap is not essential for such a state of matter to exist, but rather a finite expectation value of the pair field. Consequently, the specific probe to study the superconducting state is Cooper pair tunneling. Important progress in the understanding of pairing in atomic nuclei may arise from the systematic study of two-particle transfer reactions. Although this subject of research started about the time of the BCS papers, the quantitative calculation of absolute cross sections taking properly into account the full non-locality of the Cooper pairs (correlation length much larger than nuclear dimensions) is still an open question.

\section{Second order DWBA}

In what follows we shall exemplify the workings of the closely interweaved structure-reaction formalism presented

\footnotetext{
a e-mail: gregory.potel@gmail.com
}

above to probe, through two particle transfer reactions, pairing correlations in atomic nuclei. In this section we introduce the formalism of second order DWBA (see, for example, [2], [5]) in the context of the study of the reaction $A+a(=b+2) \rightarrow B(=A+2)+b$. We will stress the need to consider the sequential transfer of the two nucleons by virtually populating states of the intermediate nuclei $f(=b+1)$ and $F(=A+1)$ in order to obtain a reliable absolute value of the cross section.

Let us assume that two nucleons coupled to angular momentum 0 in the initial nucleus $a$ are transferred into a final state of zero angular momentum in nucleus $B$. The transition amplitude is given by the integral

$$
\begin{aligned}
& 2 \sum_{\sigma_{1} \sigma_{2}} \int d \mathbf{r}_{f F} d \mathbf{r}_{A 2} d \mathbf{r}_{b 1} \chi^{(-) *}\left(\mathbf{r}_{b B}\right) \\
& \times\left[\psi^{j_{f}}\left(\mathbf{r}_{A 1}, \sigma_{1}\right) \psi^{j_{f}}\left(\mathbf{r}_{A 2}, \sigma_{2}\right)\right]_{0}^{0 *} \\
& \quad \times v\left(r_{b 1}\right) \Psi^{(+)}\left(\mathbf{r}_{a A}, \mathbf{r}_{b 1}, \mathbf{r}_{b 2}, \sigma_{1}, \sigma_{2}\right),
\end{aligned}
$$

where $\mathbf{r}_{b 1}$ is the vector which locates neutron 1 with respect to core $b, \mathbf{r}_{A 2}$ is the vector which locates neutron 2 with respect to core $A, \mathbf{r}_{f F}$ is the relative vector between cores $f$ and $F$ etc. The $\chi$ are distorted waves (continuum wavefunctions of an optical potential), and the $\psi$ are the single particle wavefunctions of the neutrons. The potential $v\left(r_{b 1}\right)$ is a single particle mean field potential which, aside from being responsible for the transfer in the post representation (see below), is also used to define the single particle wavefunctions of the neutrons in the initial state. 


\section{EPJ Web of Conferences}

If we neglect core excitations, the above expression is exact as long as $\Psi^{(+)}\left(\mathbf{r}_{a A}, \mathbf{r}_{b 1}, \mathbf{r}_{b 2}, \sigma_{1}, \sigma_{2}\right)$ is the exact wavefunction. We can instead obtain an approximation for the transfer amplitude using

$$
\begin{aligned}
& \Psi^{(+)}\left(\mathbf{r}_{a A}, \mathbf{r}_{b 1}, \mathbf{r}_{b 2}, \sigma_{1}, \sigma_{2}\right) \\
& \quad \approx \chi^{(+)}\left(\mathbf{r}_{a A}\right)\left[\psi^{j_{i 1}}\left(\mathbf{r}_{b 1}, \sigma_{1}\right) \psi^{j_{i 2}}\left(\mathbf{r}_{b 2}, \sigma_{2}\right)\right]_{0}^{0} \\
& \quad+\sum_{K, M} \mathcal{U}_{K, M}\left(\mathbf{r}_{f F}\right)\left[\psi^{j_{f}}\left(\mathbf{r}_{A 2}, \sigma_{2}\right) \psi^{j_{i 1}}\left(\mathbf{r}_{b 1}, \sigma_{1}\right)\right]_{M}^{K}
\end{aligned}
$$

as an approximation for the incoming state. As will be shown, the first term of (2) gives rise to the simultaneous $\left(T^{(1)}\right)$ amplitude, while from second one we get the successive $\left(T_{\text {succ }}^{(2)}\right)$ and the non-orthogonality $\left(T_{N O}^{(2)}\right)$ contributions. To extract the amplitude $\mathcal{U}_{K, M}\left(\mathbf{r}_{f F}\right)$, we define $f_{K M}\left(\mathbf{r}_{f F}\right)$ as the scalar product

$$
\begin{gathered}
f_{K M}\left(\mathbf{r}_{f F}\right)=\left\langle\left[\psi^{j_{f}}\left(\mathbf{r}_{A 2}, \sigma_{2}\right) \psi^{j_{i 1}}\left(\mathbf{r}_{b 1}, \sigma_{1}\right)\right]_{M}^{K}\right| \\
\left.\Psi^{(+)}\left(\mathbf{r}_{a A}, \mathbf{r}_{b 1}, \mathbf{r}_{b 2}, \sigma_{1}, \sigma_{2}\right)\right\rangle
\end{gathered}
$$

for fixed $\mathbf{r}_{f F}$, which can be seen to obey the equation

$$
\begin{aligned}
&\left(\frac{\hbar^{2}}{2 \mu_{f F}} k_{f F}^{2}+\right.\left.\frac{\hbar^{2}}{2 \mu_{f F}} \nabla_{r_{f F}}^{2}-U\left(r_{f F}\right)\right) f_{K M}\left(\mathbf{r}_{f F}\right) \\
&=\left\langle\left[\psi^{j_{f}}\left(\mathbf{r}_{A 2}, \sigma_{2}\right) \psi^{j_{i 1}}\left(\mathbf{r}_{b 1}, \sigma_{1}\right)\right]_{M}^{K}\right| \\
& v\left(r_{c 2}\right)\left|\Psi^{(+)}\left(\mathbf{r}_{a A}, \mathbf{r}_{b 1}, \mathbf{r}_{b 2}, \sigma_{1}, \sigma_{2}\right)\right\rangle .
\end{aligned}
$$

The solution can be written in terms of the Green function $G\left(\mathbf{r}_{f F}, \mathbf{r}_{f F}^{\prime}\right)$ defined by

$$
\begin{aligned}
\left(\frac{\hbar^{2}}{2 \mu_{f F}} k_{f F}^{2}+\frac{\hbar^{2}}{2 \mu_{f F}} \nabla_{r_{f F}}^{2}-U\left(r_{f F}\right)\right) G\left(\mathbf{r}_{f F}, \mathbf{r}_{f F}^{\prime}\right) \\
=\frac{\hbar^{2}}{2 \mu_{f F}} \delta\left(\mathbf{r}_{f F}-\mathbf{r}_{f F}^{\prime}\right) .
\end{aligned}
$$

Thus,

$$
\begin{aligned}
& f_{K M}\left(\mathbf{r}_{f F}\right)= \frac{2 \mu_{f F}}{\hbar^{2}} \int d \mathbf{r}_{f F}^{\prime} G\left(\mathbf{r}_{f F}, \mathbf{r}_{f F}^{\prime}\right) \\
& \times\left\langle\left[\psi^{j_{f}}\left(\mathbf{r}_{A 2}^{\prime}, \sigma_{2}^{\prime}\right) \psi^{j_{i 1}}\left(\mathbf{r}_{b 1}^{\prime}, \sigma_{1}^{\prime}\right)\right]_{M}^{K}\right| \\
& v\left(r_{C 2}\right)\left|\Psi^{(+)}\left(\mathbf{r}_{a A}^{\prime}, \mathbf{r}_{b 1}^{\prime}, \mathbf{r}_{b 2}^{\prime}, \sigma_{1}^{\prime}, \sigma_{2}^{\prime}\right)\right\rangle \\
& \approx \frac{2 \mu_{f F}}{\hbar^{2}} \sum_{\sigma_{1}^{\prime} \sigma_{2}^{\prime}} \int d \mathbf{r}_{f F}^{\prime} d \mathbf{r}_{A 2}^{\prime} d \mathbf{r}_{b 1}^{\prime} G\left(\mathbf{r}_{f F}, \mathbf{r}_{f F}^{\prime}\right) \\
& \times\left[\psi^{j_{f}}\left(\mathbf{r}_{A 2}^{\prime}, \sigma_{2}^{\prime}\right) \psi^{j_{i 1}}\left(\mathbf{r}_{b 1}^{\prime}, \sigma_{1}^{\prime}\right)\right]_{M}^{K *} v\left(r_{c 2}^{\prime}\right) \chi^{(+)}\left(\mathbf{r}_{a A}^{\prime}\right) \\
& \quad \times\left[\psi^{j_{i 1}}\left(\mathbf{r}_{b 1}^{\prime}, \sigma_{1}^{\prime}\right) \psi^{j_{i 2}}\left(\mathbf{r}_{b 2}^{\prime}, \sigma_{2}^{\prime}\right)\right]_{0}^{0} \\
&=\mathcal{U}_{K, M}\left(\mathbf{r}_{f F}\right)+\left\langle\left[\psi^{j_{f}}\left(\mathbf{r}_{A 2}^{\prime}, \sigma_{2}\right) \psi^{j_{i 1}}\left(\mathbf{r}_{b 1}^{\prime}, \sigma_{1}\right)\right]_{M}^{K}\right. \\
&\left.\quad \chi^{(+)}\left(\mathbf{r}_{a A}^{\prime}\right)\left[\psi^{j_{i 1}}\left(\mathbf{r}_{b 1}^{\prime}, \sigma_{1}^{\prime}\right) \psi^{j_{i 2}}\left(\mathbf{r}_{b 2}^{\prime}, \sigma_{2}^{\prime}\right)\right]_{0}^{0}\right\rangle .
\end{aligned}
$$

Therefore

$$
\begin{gathered}
\mathcal{U}_{K, M}\left(\mathbf{r}_{f F}\right)=\frac{2 \mu_{f F}}{\hbar^{2}} \sum_{\sigma_{1}^{\prime} \sigma_{2}^{\prime}} \int d \mathbf{r}_{f F}^{\prime} d \mathbf{r}_{A 2}^{\prime} d \mathbf{r}_{b 1}^{\prime} G\left(\mathbf{r}_{f F}, \mathbf{r}_{f F}^{\prime}\right) \\
\times\left[\psi^{j_{f}}\left(\mathbf{r}_{A 2}^{\prime}, \sigma_{2}^{\prime}\right) \psi^{j_{i 1}}\left(\mathbf{r}_{b 1}^{\prime}, \sigma_{1}^{\prime}\right)\right]_{M}^{K *} \times v\left(r_{c 2}^{\prime}\right) \chi^{(+)}\left(\mathbf{r}_{a A}^{\prime}\right) \\
\times\left[\psi^{j_{i 1}}\left(\mathbf{r}_{b 1}^{\prime}, \sigma_{1}^{\prime}\right) \psi^{j_{i 2}}\left(\mathbf{r}_{b 2}^{\prime}, \sigma_{2}^{\prime}\right)\right]_{0}^{0}-\left\langle\left[\psi^{j_{f}}\left(\mathbf{r}_{A 2}^{\prime}, \sigma_{2}\right) \psi^{j_{i 1}}\left(\mathbf{r}_{b 1}^{\prime}, \sigma_{1}\right)\right]_{M}^{K}\right. \\
\left.\chi^{(+)}\left(\mathbf{r}_{a A}^{\prime}\right)\left[\psi^{j_{i 1}}\left(\mathbf{r}_{b 1}^{\prime}, \sigma_{1}^{\prime}\right) \psi^{j_{i 2}}\left(\mathbf{r}_{b 2}^{\prime}, \sigma_{2}^{\prime}\right)\right]_{0}^{0}\right\rangle
\end{gathered}
$$

When we substitute $\mathcal{U}_{K, M}\left(\mathbf{r}_{f F}\right)$ into (2) and (1), the first term gives rise to the successive $\left(T_{\text {succ }}^{(2)}\right)$ amplitude for the two-particle transfer, while the second term is responsible for the non-orthogonal $\left(T_{N O}^{(2)}\right)$ contribution. Explicitly,

$$
\begin{aligned}
& T^{(1)}\left(j_{i}, j_{f}\right)=2 \sum_{\sigma_{1} \sigma_{2}} \int d \mathbf{r}_{f F} d \mathbf{r}_{b 1} d \mathbf{r}_{A 2} \\
& \quad \times\left[\Psi^{j_{f}}\left(\mathbf{r}_{A 1}, \sigma_{1}\right) \Psi^{j_{f}}\left(\mathbf{r}_{A 2}, \sigma_{2}\right)\right]_{0}^{0 *} \chi_{b B}^{(-) *}\left(\mathbf{r}_{b B}\right) \\
& \quad \times v\left(\mathbf{r}_{b 1}\right)\left[\Psi^{j_{i}}\left(\mathbf{r}_{b 1}, \sigma_{1}\right) \Psi^{j_{i}}\left(\mathbf{r}_{b 2}, \sigma_{2}\right)\right]_{0}^{0} \chi_{a A}^{(+)}\left(\mathbf{r}_{a A}\right),
\end{aligned}
$$

$$
\begin{aligned}
& T_{s u c c}^{(2)}\left(j_{i}, j_{f}\right)=2 \sum_{K, M} \sum_{\sigma_{\sigma_{1} \sigma_{2}}^{\prime}} \int d \mathbf{r}_{f F} d \mathbf{r}_{b 1} d \mathbf{r}_{A 2} \\
& \times\left[\Psi^{j_{f}}\left(\mathbf{r}_{A 1}, \sigma_{1}\right) \Psi^{j_{f}}\left(\mathbf{r}_{A 2}, \sigma_{2}\right)\right]_{0}^{0 *} \chi_{b B}^{(-) *}\left(\mathbf{r}_{b B}\right) v\left(\mathbf{r}_{b 1}\right) \\
& \times\left[\Psi^{j_{f}}\left(\mathbf{r}_{A 2}, \sigma_{2}\right) \Psi^{j_{i}}\left(\mathbf{r}_{b 1}, \sigma_{1}\right)\right]_{M}^{K} \int d \mathbf{r}_{f F}^{\prime} d \mathbf{r}_{b 1}^{\prime} d \mathbf{r}_{A 2}^{\prime} G\left(\mathbf{r}_{f F}, \mathbf{r}_{f F}^{\prime}\right) \\
& \times\left[\Psi^{j_{f}}\left(\mathbf{r}_{A 2}^{\prime}, \sigma_{2}^{\prime}\right) \Psi^{j_{i}}\left(\mathbf{r}_{b 1}^{\prime}, \sigma_{1}^{\prime}\right)\right]_{M}^{K} \frac{2 \mu_{f F}}{\hbar^{2}} v\left(\mathbf{r}_{f 2}^{\prime}\right) \\
& \times\left[\Psi^{j_{i}}\left(\mathbf{r}_{A 2}^{\prime}, \sigma_{2}^{\prime}\right) \Psi^{j_{i}}\left(\mathbf{r}_{b 1}^{\prime}, \sigma_{1}^{\prime}\right)\right]_{0}^{0} \chi_{a A}^{(+)}\left(\mathbf{r}_{a A}^{\prime}\right), \quad(8 \mathrm{~b}) \\
& T_{N O}^{(2)}\left(j_{i}, j_{f}\right)=2 \sum_{K, M} \sum_{\sigma_{1} \sigma_{2}} \int d \mathbf{r}_{f F} d \mathbf{r}_{b 1} d \mathbf{r}_{A 2} \\
& \times\left[\Psi^{j_{f}}\left(\mathbf{r}_{A 1}, \sigma_{1}\right) \Psi^{j_{f}}\left(\mathbf{r}_{A 2}, \sigma_{2}\right)\right]_{0}^{0 *} \chi_{b B}^{(-) *}\left(\mathbf{r}_{b B}\right) v\left(\mathbf{r}_{b 1}\right) \\
& \times\left[\Psi^{j_{f}}\left(\mathbf{r}_{A 2}, \sigma_{2}\right) \Psi^{j_{i}}\left(\mathbf{r}_{b 1}, \sigma_{1}\right)\right]_{M}^{K} \int d \mathbf{r}_{b 1}^{\prime} d \mathbf{r}_{A 2}^{\prime} \\
& \quad \times\left[\Psi^{j_{f}}\left(\mathbf{r}_{A 2}^{\prime}, \sigma_{2}^{\prime}\right) \Psi^{j_{i}}\left(\mathbf{r}_{b 1}^{\prime}, \sigma_{1}^{\prime}\right)\right]_{M}^{K} \\
& \quad \times\left[\Psi^{j_{i}}\left(\mathbf{r}_{A 2}^{\prime}, \sigma_{2}^{\prime}\right) \Psi^{j_{i}}\left(\mathbf{r}_{b 1}^{\prime}, \sigma_{1}^{\prime}\right)\right]_{0}^{0} \chi_{a A}^{(+)}\left(\mathbf{r}_{a A}^{\prime}\right) . \quad(8 \mathrm{c})
\end{aligned}
$$

Remember that in these expressions, the spatial and spin coordinates of the two transferred nucleons are explicitly referred to with the subscripts 1 and 2 . The subscripts $A$ and $b$ indicate the core to which the position of each of the nucleons are referred to. The vectors $\mathbf{r}_{a A}, \mathbf{r}_{b B}$ and $\mathbf{r}_{f F}$ are the relative motion coordinates in the initial, final and intermediate channels respectively. The transition potential responsible for the transfer of the pair is, in the post representation,

$$
V_{\beta}=v_{b B}-U_{\beta},
$$

where $v_{b B}$ is the interaction between the nuclei $B$ and $b$, and $U_{\beta}$ is the optical potential in the final channel. We make the 
assumption that $v_{b B}$ can be decomposed into a term containing the interaction between the cores $A$ and $b$ and the potential describing the interaction between $b$ and each of the transferred nucleons, namely

$$
v_{b B}=v_{b A}+v_{b 1}+v_{b 2},
$$

where $v_{b 1}$ and $v_{b 2}$ is the same mean field potential we have used to define the single-particle wavefunctions of the neutrons in the nucleus $a$. The transition potential is

$$
V_{\beta}=v_{b A}+v_{b 1}+v_{b 2}-U_{\beta} .
$$

Assuming that $\left\langle\beta\left|v_{b A}\right| \alpha\right\rangle \simeq\left\langle\beta\left|U_{\beta}\right| \alpha\right\rangle$ (i.e, assuming that the matrix element of the core-core interaction between the initial and final states is very similar to the matrix element of the real part of the optical potential), one obtains the final expression of the transfer potential in the post representation,

$$
V_{\beta} \simeq v_{b 1}+v_{b 2} \text {. }
$$

This last approximation seems reasonable when dealing with heavy ion reactions in which there is no charge transfer, but more care has to be exerted when dealing with reactions in which light ions are involved.

To calculate the total pair transfer amplitude, a sum of the contributions associated with each mean field contribution, labeled by the quantum numbers $\left(j_{i}, j_{f}\right)$ and weighted with the correspondent two-nucleon spectroscopic amplitude $B_{j}$, is to be carried out leading to

$$
\begin{aligned}
T_{2 N T}=\sum_{j_{f} j_{i}} B_{j_{f}} B_{j_{i}}( & T^{(1)}\left(j_{i}, j_{f}\right) \\
& \left.+T_{\text {succ }}^{(2)}\left(j_{i}, j_{f}\right)-T_{N O}^{(2)}\left(j_{i}, j_{f}\right)\right) .
\end{aligned}
$$

The quantity $B_{j} \equiv B(j=0 ; j, j)$ is a special realization of the two-nucleon spectroscopy amplitude

$$
\begin{aligned}
B\left(J ; j_{1}, j_{2}\right)=\sum_{M, M_{i}} & \left\langle J_{i} M_{i} J M \mid J_{f} M_{f}\right\rangle \\
& \times\left\langle\Psi_{J_{f} M_{f}}\left|P^{\dagger}\left(j_{1}, j_{2} ; J M\right)\right| \Psi_{J_{i} M_{i}}\right\rangle
\end{aligned}
$$

where

$$
\begin{aligned}
& P^{\dagger}\left(j_{1}, j_{2} ; J M\right) \\
& \quad=N \sum_{m}\left\langle j_{1} m j_{2} M-m \mid J M\right\rangle a_{j_{1} m}^{\dagger} a_{j_{2} M-m}^{\dagger},
\end{aligned}
$$

is the (renormalized) pair creation operator. In other words, $B\left(J ; j_{1}, j_{2}\right)$ is the amplitude of finding in the $\left|A+2 ; J_{f}, M_{f}\right\rangle$ nuclear state, two nucleons moving in the single-particle orbitals $j_{1}$ and $j_{2}$ and coupled to angular momentum $J$, on top of the state $\left|A ; J_{i}, M_{i}\right\rangle$, coupled to total angular momentum $\left(J, J_{i}\right) J$. Of notice that in Eq. (13) the nuclear structure information which is essentially all contained in the amplitudes $B_{i j}$, is closely interweaved with the reaction amplitudes. This is the reason why the absolute value of twonucleon transfer cross sections can display large enhancements as compared to pure configuration cross sections, thus revealing the coherence of (Cooper) pair correlations resulting from the pairing interaction. Eq. (13) also testifies to the fact that quantitatively accurate description of pair transfer requires to treat on par both structure and reaction aspects of the process. Within this scenario Eq. (13) provides another circumstantial evidence strongly supporting the fact that structure and reactions are but two aspects of the same many-body physics.

The differential cross section associated with the twoparticle transfer amplitudes discussed above can be written as

$$
\frac{d \sigma}{d \Omega}=\frac{\mu_{i} \mu_{f}}{\left(4 \pi \hbar^{2}\right)^{2}} \frac{k_{f}}{k_{i}}\left|T_{2 N T}\right|^{2},
$$

where $\mu_{i}, \mu_{f}$ are the reduced masses in entrance and exit channels respectively, while $k_{f}, k_{i}$ are the corresponding relative momenta.

Note that in this approach the interaction potential $v(\mathbf{r})$ responsible for the transfer is of single particle nature. As a two-particle transfer reaction is a process in which two nucleon change state, it is of (at least) second order in perturbation theory. It is then not surprising that the nonorthogonal amplitude tend to cancel the simultaneous transfer contribution, which is only a spurious consequence of the fact that the initial and final states are described with non-orthogonal wavefunctions. This cancelation is exact if the number of intermediate states form a complete basis of the two-particle Hilbert states. A numerical approximate realization of this cancelation is shown in Fig. 1, where we show the results of the analysis of the ${ }^{132} \operatorname{Sn}(p, t)^{130} \mathrm{Sn}$ reaction at a laboratory energy of $20 \mathrm{MeV}$. It can be seen that the two-neutron transfer reaction is essentially a sequential (successive) process.

After some manipulation (see also [2]), we obtain a form for the successive amplitude (8b) which can be implemented in a computer to be numerically evaluated:

$$
\begin{aligned}
T_{2 N T}^{V V}= & \frac{1024 \mu_{C c} \pi^{9 / 2} i}{\hbar^{2} k_{A a} k_{B b} k_{C c}} \frac{1}{\sqrt{\left(2 j_{i}+1\right)\left(2 j_{f}+1\right)}} \\
\times & \sum_{K} \frac{1}{2 K+1}\left(\left(l_{f} \frac{1}{2}\right)_{j_{f}}\left(l_{i} \frac{1}{2}\right)_{j_{i}} \mid\left(l_{f} l_{i}\right)_{K}\left(\frac{1}{2} \frac{1}{2}\right)_{0}\right)_{K}^{2} \\
& \times \sum_{l_{c}, l} e^{i\left(\sigma_{i}^{l}+\sigma_{f}^{l}\right)} \frac{\left(2 l_{c}+1\right)}{\sqrt{2 l+1}} Y_{0}^{l}\left(\hat{k}_{B b}\right) S_{K, l, l_{c}},
\end{aligned}
$$

with

$$
\begin{aligned}
& S_{K, l, l_{c}}= \int r_{C c}^{2} d r_{C c} r_{b 1}^{2} d r_{b 1} \sin \theta d \theta v\left(r_{b 1}\right) \\
& \times u_{l_{f}}\left(r_{C 1}\right) u_{l_{i}}\left(r_{b 1}\right) \frac{s_{K, l, l_{c}}\left(r_{C c}\right)}{r_{C c}} \frac{F_{l}\left(r_{B b}\right)}{r_{B b}} \\
& \times \sum_{M}\left\langle l_{c} 0 l M \mid K M\right\rangle\left[Y^{l_{f}}\left(\hat{r}_{C 1}\right) Y^{l_{i}}(\theta+\pi, 0)\right]_{M}^{K} Y_{M}^{l^{*}}\left(\hat{r}_{B b}\right),
\end{aligned}
$$




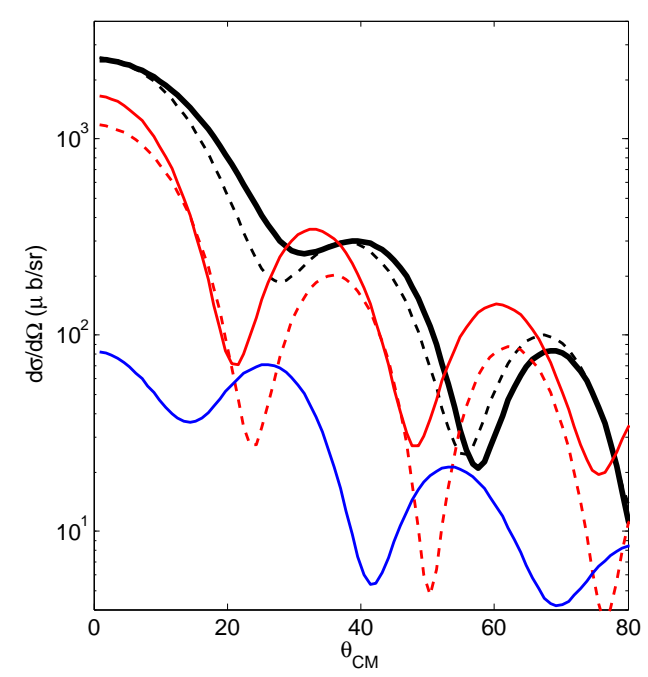

Fig. 1. Contributions to the total two-neutron transfer cross section (thick black line) of the different amplitudes $(8 \mathrm{a}, 8 \mathrm{~b}, 8 \mathrm{c})$, for the ${ }^{132} \mathrm{Sn}(p, t)^{130} \mathrm{Sn}$ reaction at a laboratory energy of $20 \mathrm{MeV}$. Note that the simultaneous (dashed red line) and non-orthogonal (red line) contributions are in anti-phase, so that the contribution corresponding to the coherent superposition of these two amplitudes (blue line) tend to cancel. The calculated total cross section thus essentially coincides with the successive (dashed black line) process.

and

$$
\begin{aligned}
& s_{K, l, l_{c}}\left(r_{C c}\right)=\int r_{C c}^{\prime 2} d r_{C c}^{\prime} r_{A 2}^{\prime 2} d r_{A 2}^{\prime} \sin \theta^{\prime} d \theta^{\prime} v\left(r_{c 2}^{\prime}\right) \\
& \times u_{l_{f}}\left(r_{A 2}^{\prime}\right) u_{l_{i}}\left(r_{c 2}^{\prime}\right) \frac{F_{l}\left(r_{A a}^{\prime}\right)}{r_{A a}^{\prime}} \frac{f_{l_{c}}\left(k_{C c}, r_{<}\right) P_{l_{c}}\left(k_{C c}, r_{>}\right)}{r_{C c}^{\prime}} \\
& \times \sum_{M}\left\langle l_{c} 0 l M \mid K M\right\rangle\left[Y^{l_{f}}\left(\hat{r}_{A 2}^{\prime}\right) Y^{l_{i}}\left(\hat{r}_{c 2}^{\prime}\right)\right]_{M}^{K *} Y_{M}^{l}\left(\hat{r}_{A a}^{\prime}\right) .
\end{aligned}
$$

\section{The $p\left({ }^{11} \mathrm{Li},{ }^{9} \mathrm{Li}\right) t$ reaction: pairing in exotic halo light nuclei}

As a revealing example of the kind of analysis that can be carried out within the framework described above, we will consider the $p\left({ }^{11} \mathrm{Li},{ }^{9} \mathrm{Li}\right) t$ reaction induced by the exotic halo nucleus ${ }^{11} \mathrm{Li}$ ([9], [14]).

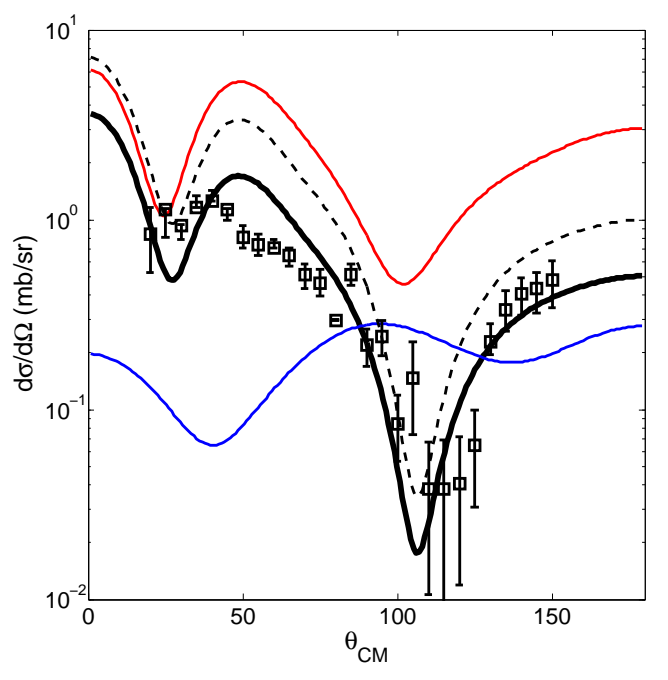

Fig. 2. Experimental ([9]) and theoretical (thick black line) differential cross sections of the $p\left({ }^{11} \mathrm{Li},{ }^{9} \mathrm{Li}\right) t$ reaction at a laboratory energy of $33 \mathrm{MeV}$ ([14]). We also show the results obtained without coupling with collective states (dashed black line), and with two different pure single particle configurations of the twoneutron halo: $\left(s_{1 / 2}\right)^{2}$ (red line) and $\left(p_{1 / 2}\right)^{2}$ (blue line). The optical potentials used are from [1] and [9].

There exists conspicuous circumstantial evidence which testifies to the important role medium polarization effects play in the phenomenon of nuclear superfluidity (see e.g. [4] and refs. therein). In spite of this, a quantitative assessment of it is still lacking. Specially promising in this quest are highly polarizable exotic nuclei, in particular, the light halo nucleus ${ }^{11} \mathrm{Li}$, for which, the balance between bare and induced pairing interactions is strongly shifted in favour of the induced interaction ([7], see also [10], [13], [12]).

In this nucleus, the last two neutrons are very weakly bound ( $S_{2 n} \approx 380 \mathrm{keV} \mathrm{[6],} \mathrm{[11],} \mathrm{[16]).} \mathrm{If} \mathrm{one} \mathrm{neutron} \mathrm{is}$ taken away from ${ }^{11} \mathrm{Li}$, a second neutron will come out immediately leaving behind the core of the system, the ordinary nucleus ${ }^{9} \mathrm{Li}$. This result testifies to the fact that pairing is central in the stability of ${ }^{11} \mathrm{Li}$ (see e.g. [3], [8]).

In ref. [7] it has been shown that the two outer (halo) neutrons of ${ }^{11} \mathrm{Li}$ in its ground state attract each other, not only due to the strong nuclear force acting among them, but also and primarily due to the virtual processes associated with the exchange of collective vibrations. In particular, 
the quadrupole vibration of the ${ }^{9} \mathrm{Li}$ core, and the dipole vibration associated with the neutron halo field (pigmy resonance of ${ }^{11} \mathrm{Li}$ [15]). Such a pairing mechanism is clearly reflected in the calculated ground state wavefunction of ${ }^{11} \mathrm{Li}$ [7],

$$
\left|{ }^{11} \operatorname{Li}(g s) ; 3 / 2^{-}\right\rangle=|\tilde{0}\rangle_{v} \otimes\left|1 p_{3 / 2}(\pi)\right\rangle,
$$

where $\pi$ and $v$ indicate proton and neutron degrees of freedom respectively, while $|\tilde{0}\rangle_{v}$ indicates the halo neutron Cooper pair wavefunction, that is,

$$
\begin{aligned}
|\tilde{0}\rangle_{v}=|0\rangle+\alpha \mid & \left.\left(p_{1 / 2}, s_{1 / 2}\right)_{1^{-}} \otimes 1^{-} ; 0\right\rangle \\
& +\beta\left|\left(s_{1 / 2}, d_{5 / 2}\right)_{2^{+}} \otimes 2^{+} ; 0\right\rangle,
\end{aligned}
$$

with

$$
\alpha \approx 0.7, \quad \text { and } \quad \beta \approx 0.1,
$$

and

$$
|0\rangle=0.45\left|s_{1 / 2}^{2}(0)\right\rangle+0.55\left|p_{1 / 2}^{2}(0)\right\rangle+0.04\left|d_{5 / 2}^{2}(0)\right\rangle,
$$

the states $\left|1^{-}\right\rangle$and $\left|2^{+}\right\rangle$being the (RPA) states describing the dipole pigmy resonance of ${ }^{11} \mathrm{Li}$ and the quadrupole vibration of the core ${ }^{9} \mathrm{Li}$ (see [7], see also Tables 11.3 and 11.5 of ref [4]). Note that in this model half of the wavefunction of the ground state of ${ }^{11} \mathrm{Li}$ correspond to states of the halo coupled to collective excited states of the system. In Fig. 2 we show the results of the experimental and theoretical differential cross sections of the $p\left({ }^{11} \mathrm{Li},{ }^{9} \mathrm{Li}\right) t$ reaction with a $33 \mathrm{MeV}$ lithium beam ([9], [14]). We compare the predictions obtained within the structure model described above with other calculations in which we neglect ground state correlations (coupling to collective states), and in which we describe the neutron halo as single a particle configuration.

\section{Conclusions}

As it emerges from the previous narrative, theoretical predictions reproduce the data within experimental errors without free parameters. This is a consequence of the use of reliable optical parameters for entrance, intermediate and exit channels and to the treatment, on equal footing, of the structure and of the reaction aspects of the phenomena under discussion. Within this scenario, it is only a question of time before the optical potential becomes routine part of the reaction-structure computational output/input.

It is well established that single Cooper pair transfer is the specific tool to probe pairing correlations in nuclei. This fact translates itself through structure-reaction calculations, in the fact that the absolute value of two-particle transfer cross sections is the result of the interweaving of a number of structure amplitudes and of single-particle reaction form factors.

Financial support from the Ministry of Science and Innovation of Spain grants FPA2009-07653 and ACI20091056 are acknowledged by FB and GP and by FB respectively.

\section{References}

1. H. An and C. Cai, Global deuteron optical model potential for the energy range up to $183 \mathrm{MeV}$, Phys. Rev. C 73 (2006), 054605.

2. B. F. Bayman and J. Chen, One-step and two-step contributions to two-nucleon transfer reactions, Phys. Rev. C 26 (1982), 1509.

3. G. F. Bertsch and H. Esbensen, Pair correlations near the neutron drip line, Annals of Physics 209 (1991).

4. D. Brink and R. A. Broglia, Nuclear superfluidity, Cambridge University Press, Cambridge, 2005.

5. R.A. Broglia and A. Winther, Heavy ion reactions, 2 nd ed., Westview Press, Perseus Books, Boulder, 2005.

6. C. Bachelet et al., New Binding Energy for the TwoNeutron Halo of ${ }^{11} \mathrm{Li}$, Phys. Rev. Lett. 100 (2008), 182501.

7. F. Barranco et al., The halo of the exotic nucleus ${ }^{11} \mathrm{Li}$ : a single Cooper pair, Europ. Phys. J. A 11 (2001), 385.

8. K. Hagino and H. Sagawa, Pairing correlations in $n u-$ clei on the neutron-drip line, Phys. Rev. C 72 (2005), 044321.

9. I. Tanihata et al., Measurement of the two-halo neutron transfer reaction ${ }^{1} \mathrm{H}\left({ }^{11} \mathrm{Li},{ }^{9} \mathrm{Li}\right)^{3} \mathrm{H}$ at $3 \mathrm{~A} \mathrm{MeV}$, Phys. Rev. Lett. 100 (2008), 192502.

10. K. Hagino et al., Coexistence of BCS- and BEC-like pair structures in halo nuclei, Phys. Rev. Lett. 99 (2007), 022506.

11. M. Smith et al., First penning-trap mass measurement of the exotic halo nucleus ${ }^{11} \mathrm{Li}$, Phys. Rev. Lett. 101 (2008), 202501.

12. N. Vinh Mau and J. C. Pacheco, Structure of the ${ }^{11} \mathrm{Li}$ nucleus, Nucl. Phys. A 607 (1996), 163.

13. F.M. Nunes, Valence pairing, core deformation and the development of two-neutron halos, Nucl. Phys. A 757 (2005), 349.

14. G. Potel, F. Barranco, E. Vigezzi, and R. A. Broglia, Evidence for phonon mediated pairing interaction in the halo of the nucleus ${ }^{11} \mathrm{Li}$, Phys. Rev. Lett. 105 (2010), 172502.

15. T. Nakamura et al., Observation of Strong Low-Lying E1 Strength in the Two-Neutron Halo Nucleus ${ }^{11} \mathrm{Li}$, Phys. Rev. Lett. 96 (2006), 252502.

16. T. Roger et al., Mass of ${ }^{11} \mathrm{Li}$ from the ${ }^{1} \mathrm{H}\left({ }^{11} \mathrm{Li},{ }^{9} \mathrm{Li}\right)^{3} \mathrm{H}$ reaction, Phys. Rev.C 79 (2009), 031603. 